\title{
Design of New Concatenated Space-Time Block Codes Using Odd Transmit Antennas
}

\author{
Taejin Jung ${ }^{1}$ and Wangrok $\mathrm{Oh}^{2, \star}$ \\ ${ }^{1}$ Dept. of Electronics and Computer Eng., Chonnam National University, \\ 300 Yongbong-dong, Puk-gu, Kwangju, 500-757, Korea \\ tjjung@chonnam.ac.kr \\ 2 Div. of Electrical and Computer Eng., Chungnam National University, \\ 220 Gung-dong, Yuseong-gu, Daejeon, 305-764, Korea \\ kingrock@cnu.ac.kr
}

\begin{abstract}
In this paper, a new class of space-time block codes achieving full-rate and full spatial diversity for QAM is proposed when using any odd transmit antennas over quasi-static Rayleigh fading channels. Like the conventional A-ST-CR codes [10], the proposed codes are constructed by serially concatenating the constellation-rotating precoders [7]-[9] with the Alamouti scheme [3]. Computer simulations show that for the case of QPSK, the best code in this class achieves approximately 1.5dB larger coding gain than the existing ST-CR code [8], [9] for both 3 and 5 transmit antennas at average $\mathrm{SER}=10^{-5}$ and for the case of 16 -QAM, $3 \mathrm{~dB}$ for 3 transmit antennas. The codes possessing quasi-orthogonal characteristic are also included in this class, allowing simple ML decoding with virtually no performance loss compared to the best code in the class.
\end{abstract}

\section{Introduction}

Recently, the space-time coding technique [1] using multiple transmit antennas has received considerable attention as a promising technique to enhance the capacity and quality of mobile wireless systems. Tarokh et al. in [2] developed orthogonal space-time block codes (O-STBCs) based on orthogonal designs achieving full diversity and allowing simple maximum likelihood (ML) decoding. Unfortunately, full-rate O-STBCs for general complex modulation such as PSK and QAM do not exist when the number of transmit antennas is larger than two [2], [3]. Yan et al. in [8], [9] proposed so called space-time constellation-rotating (ST-CR) codes achieving both full-rate and full spatial diversity for general QAM when using any number of transmit antennas. This is done by transmitting the precoded symbols generated by multiplying a vector of QAM symbols via linear constellation-rotating precoders. By serially concatenating these linear precoders with the Alamouti scheme [3], Jung et al. in [10] presented so called Alamouti ST-CR (A-ST-CR) codes enjoying larger coding gains than the ST-CR codes without any loss of code rate. However, these codes were only designed for an

\footnotetext{
^ Corresponding author.
} 
even number of transmit antennas. Also, these two classes of codes based on the linear precoders have a great deficiency of not satisfying the Tarokh's orthogonal designs [2], resulting in a greatly higher ML decoding complexity compared to the O-STBCs.

Hence, based on the design idea in the A-ST-CR codes, we will present a new class of STBCs achieving full rate and full diversity for QAM and quasi-static Rayleigh fading channels when using any odd number of transmit antennas. These codes are designed by serially concatenating the constellation-rotating precoders with the Alamouti scheme like the conventional A-ST-CR codes. Computer simulations show that for the case of QPSK, the best code in this class achieves approximately $1.5 \mathrm{~dB}$ larger coding gain than the existing ST-CR code for both 3 and 5 transmit antennas at average $\mathrm{SER}=10^{-5}$ and for the case of 16-QAM, 3dB for 3 transmit antennas. Specifically, new codes satisfying quasiorthogonal characteristic [4]-[6] are also included in this class, exhibiting almost same error performance as the best code in the class. The quasi-orthogonal property allows a ML decoder at the receiver to decode two groups of modulated symbols separately, resulting in greatly simplified ML decoding at the receiver. The simple ML decoding algorithm based on the quasi-orthogonal characteristic will be presented in Section IV in detail.

This paper is organized as follows. In Section II, system model considered in this paper is described and in Section III, some important characteristics of the conventional ST-CR and A-ST-CR codes are briefly reviewed. Then we design the new full-rate STBCs with full diversity for odd transmit antennas in Section III and present the simulation results for these codes in Section IV. Finally, conclusions are drawn in Section V.

\section{System Model}

The basic system model considered in this paper is identical to that of STBC with $N$ transmit and one receive antennas under quasi-static Rayleigh fading channels [10], which is depicted in Fig. 1.

The transmitter first groups the QAM symbols with unit energy to form vectors of length $L, \mathbf{x}=\left[x_{1}, \cdots, x_{L}\right]^{T}$ where $\mathbf{z}^{T}$ denotes the transpose vector of $\mathbf{z}$. This vector is then input to the space-time encoder to form a codeword matrix $\mathbf{S}(\mathbf{x})=\left\{s_{i j}\right\}$ of size $T_{0} \times N$. The codeword symbol $s_{i j}$ is then transmitted on antenna $j$ at time $i$. Here, we focus on STBCs achieving full rate by setting $T_{0}=L$. We also normalize the codeword matrix $\mathbf{S}(\mathbf{x})$ with energy constraint $E\left\{\|\mathbf{S}(\mathbf{x})\|^{2}\right\}=L$ where $E\{\cdot\}$ and $\|\cdot\|$ denote the expectation operator and Frobenius norm, respectively.

The symbols transmitted from different transmit antennas are assumed to experience independent Rayleigh fading. The channel is also assumed to be quasistatic in the sense that the channel do not vary significantly during the transmission of the code matrix. Hence, a received vector $\mathbf{y}=\left[y_{1}, \cdots, y_{L}\right]^{T}$ with a matched filter output $y_{i}$ at time $i$ is given as

$$
\mathbf{y}=\sqrt{E_{s}} \mathbf{S}(\mathbf{x}) \mathbf{h}+\mathbf{n}
$$




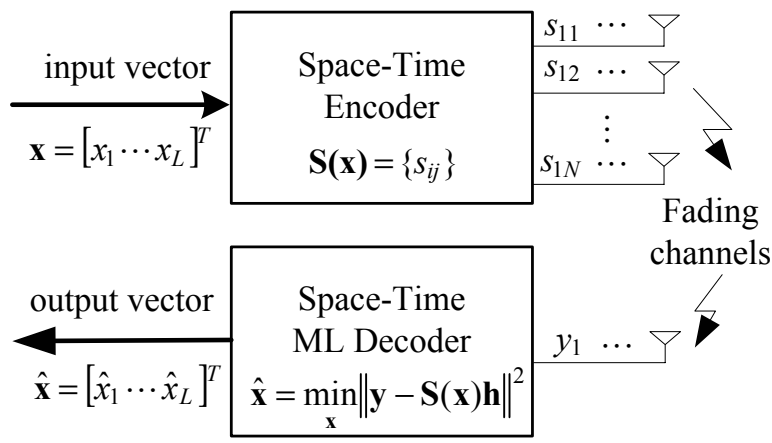

Fig. 1. System model for $\mathrm{STBC}$ with $N$ transmit and one receive antennas

where $E_{s}$ and $\mathbf{h}=\left[h_{1}, \cdots, h_{N}\right]^{T}$ denote the average received symbol energy and the channel gain vector, respectively, where $h_{n}$ represents the complex channel gain between the $n$th transmit antenna and the receive antenna with zero mean and unit variance. Also, $\mathbf{n}=\left[n_{1}, \cdots, n_{L}\right]^{T}$ denotes the received noise vector of length $L$ where $n_{i}$ represents a sample of the i.i.d. complex Gaussian random variable at time $i$ with zero mean and variance $N_{0}$.

It is assumed that the channel gain vector is perfectly known at the receiver. Using this assumption, ML decoding is performed at the receiver by choosing $\hat{\mathbf{x}}$ such that $\mathbf{S}(\hat{\mathbf{x}}) \mathbf{h}$ is closest to $\mathbf{y}$ in terms of Euclidean distance given as

$$
\hat{\mathbf{x}}=\arg \min _{\mathbf{x}}\|\mathbf{y}-\mathbf{S}(\mathbf{x}) \mathbf{h}\| .
$$

\section{Conventional ST-CR and A-ST-CR Codes}

Both the conventional ST-CR [8], [9] and A-ST-CR [10] encoders with even $N$ transmit antennas first generate a precoded vector $\mathbf{r}=\left[r_{1}, \cdots, r_{N}\right]^{T}$ of length $N$ by multiplying an $N$ dimensional input vector $\mathbf{x}$ of length $N$ by a constellationrotating precoder $\boldsymbol{\Theta}$ of size $N \times N$, i.e., $\mathbf{r}=\boldsymbol{\Theta} \mathbf{x}$. The ST-CR encoder transmits $r_{i}$ at time $i$ using a subgroup of the $N$ transmit antennas so as to guarantee that the symbol $r_{i}$ experiences independent fading. But, the A-ST-CR encoder groups the $N$ rotated symbols into $N / 2$ symbol pairs which are then encoded by the Alamouti encoder [3] and transmitted on $N / 2$ antenna pairs in a timemultiplexed fashion. Examples of the such codes are

$$
\mathbf{S}(\mathbf{r})=\left\{\begin{array}{l}
\operatorname{diag}\left(r_{1}, \cdots, r_{N}\right), \mathrm{ST}-\mathrm{CR} \\
\frac{1}{\sqrt{2}} \operatorname{diag}\left(\mathbf{S}_{\mathrm{A}}\left(r_{1}, r_{2}\right), \cdots, \mathbf{S}_{\mathrm{A}}\left(r_{N-1}, r_{N}\right)\right), \text { A-ST-CR }
\end{array}\right.
$$

where $\mathbf{S}_{\mathrm{A}}(a, b) \triangleq\left[\begin{array}{cc}a & b \\ -b^{*} & a^{*}\end{array}\right]$ denotes the Alamouti codeword [3]. For both codes, the determinants of the $N \times N$ matrices $\mathbf{A} \triangleq \mathbf{S}\left(\mathbf{r}-\mathbf{r}^{\prime}\right)^{*} \mathbf{S}\left(\mathbf{r}-\mathbf{r}^{\prime}\right)$ for distinct input vectors $\mathbf{x}, \mathbf{x}^{\prime}$, can be easily calculated as [8]-[10] 


$$
\operatorname{det}(\mathbf{A})=\left\{\begin{array}{l}
\prod_{i=1}^{N}\left|d_{i}\right|^{2}, \mathrm{ST}-\mathrm{CR} \\
\prod_{i=1}^{N / 2} \frac{1}{4}\left(\left|d_{2 i-1}\right|^{2}+\left|d_{2 i}\right|^{2}\right)^{2}, \mathrm{~A}-\mathrm{ST}-\mathrm{CR}
\end{array}\right.
$$

with $d_{i} \triangleq r_{i}-r_{i}^{\prime}$ where $r_{i}$ and $r_{i}^{\prime}$ denote the $i$ th entries of $\mathbf{r}$ and $\mathbf{r}^{\prime}$, respectively. Also, $\mathbf{r}$ and $\mathbf{r}^{\prime}$ are the precoded vectors corresponding to $\mathbf{x}$ and $\mathbf{x}^{\prime}$, respectively.

It is noted that the linear precoders $\boldsymbol{\Theta}$ used in the ST-CR and the A-ST$\mathrm{CR}$ codes [8]-[10] are always designed so that $d_{i} \neq 0\left(\right.$ or $\left.r_{i} \neq r_{i}^{\prime}\right),{ }^{\forall} i$, for any two distinct input vectors $\mathbf{x}, \mathbf{x}^{\prime}$, referred to as the rotation property [10]. Hence, we can easily observe that the determinants of (4) are always positive due to the rotation property of $\boldsymbol{\Theta}$ and thus, both the ST-CR and the A-ST-CR codes of (3) satisfy the Tarokh's rank criterion [1], guaranteeing full spatial diversity. Even though these two codes have a same diversity order $N$, the A-ST-CR code outperforms the ST-CR code due to its improved coding gain [10].

In [7]-[9], the unitary precoders $\boldsymbol{\Theta}$ optimized in a sense of the Tarokh's determinant criterion [1] were investigated and presented by using algebraic design tools and also an exhaustive search method. Note that unitary (or orthogonal) precoders have a preferable feature of guaranteeing no performance loss in nonfading AWGN channels. This is because unitarity of precoders preserves Euclidean distance between any two constellation points. In particular, the unitary precoders $\Theta$ based on algebraic design theory are given as [8], [9]

$$
\boldsymbol{\Theta}=\left\{\begin{array}{l}
\frac{1}{\sqrt{N}} \operatorname{VDM}\left(\alpha_{0}, \alpha_{1}, \cdots, \alpha_{N-1}\right), N=2^{n} \\
\mathbf{F}_{N} \operatorname{diag}\left(1, \alpha, \cdots, \alpha^{N-1}\right), N \neq 2^{n}
\end{array}\right.
$$

where VDM and $\mathbf{F}_{N}$ denote the Vandermonde and the $N$-point inverse FFT matrices, respectively. Also, $\alpha_{i}=\exp (j 2 \pi(i+1 / 4) / N), i=0, \cdots, N-1$ and $\alpha=\exp (j 2 \pi / P)$ where $P$ is a positive integer. For the specific case of $N=3$, an optimal $\Theta$ different from (5) is presented in [8], [9]

$$
\boldsymbol{\Theta}=\left[\begin{array}{ccc}
0.687 & 0.513-0.113 j-0.428+0.264 j \\
-0.358-0.308 j & 0.696-0.172 j-0.011-0.513 j \\
0.190+0.520 j & 0.243-0.389 j & 0.696
\end{array}\right]
$$

\section{Design of New Concatenated STBCs}

In Section III, we briefly review the characteristics of the conventional A-STCR code in (3) designed by serially concatenated the linear precoders with the Alamouti scheme for even $N$ transmit antennas. In this Section, based on the design structure of this A-ST-CR code, we will present new several concatenated STBCs which can be used in systems with odd $N-1$ transmit antennas.

\subsection{New STBCs Using $\Theta$ of $N \times N$}

One of conventional methods for a STBC with $N$ transmit antennas to be used in systems with $N-1$ transmit antennas is simply to delete one of column vectors 
of the code [2]. Thus, by simply deleting the last column vector of the A-ST-CR code in (3), we may easily construct a new STBC for $N-1$ transmit antennas given as

$$
\mathbf{S}(\mathbf{r})=\sqrt{\frac{N}{2(N-1)}}\left[\begin{array}{cccc}
\mathbf{S}_{\mathrm{A}}\left(r_{1}, r_{2}\right) & \cdots & \mathbf{0}_{2} & \mathbf{0}_{2 \times 1} \\
\vdots & \ddots & \vdots & \vdots \\
\mathbf{0}_{2} & \cdots & \mathbf{S}_{\mathrm{A}}\left(r_{N-3}, r_{N-2}\right) & \mathbf{0}_{2 \times 1} \\
\mathbf{0}_{2 \times 1}^{T} & \cdots & \mathbf{0}_{2 \times 1}^{T} & r_{N-1} \\
\mathbf{0}_{2 \times 1}^{T} & \cdots & \mathbf{0}_{2 \times 1}^{T} & -r_{N}^{*}
\end{array}\right]
$$

where $\mathbf{0}_{2}$ and $\mathbf{0}_{2 \times 1}$ denote a zero matrix of size $2 \times 2$ and a zero column vector of length two, respectively. Also, $\sqrt{N /(2(N-1))}$ is a normalizing factor with the total transmitted power constraint $E\left\{\|\mathbf{S}(\mathbf{r})\|^{2}\right\}=N$. For this code, the determinant of $\mathbf{A}$ matrix of size $(N-1) \times(N-1)$ for any two distinct input vectors $\mathbf{x}, \mathbf{x}^{\prime}$ is computed as

$$
\operatorname{det}(\mathbf{A})=\left(\frac{N}{2(N-1)}\right)^{N-1}\left(\prod_{i=1}^{\frac{N}{2}-1}\left(\left|d_{2 i-1}\right|^{2}+\left|d_{2 i}\right|^{2}\right)^{2}\right)\left(\left|d_{N-1}\right|^{2}+\left|d_{N}\right|^{2}\right)(8)
$$

where $d_{i}=r_{i}-r_{i}^{\prime}$ is defined in (41). Hence, due to the rotation property of $\boldsymbol{\Theta}$, i.e., $d_{i} \neq 0,{ }^{\forall}$, we can easily know that the determinant of (8) is always positive and thus, the code of (7) achieves full spatial diversity like the conventional ST$\mathrm{CR}$ and A-ST-CR codes. The new code is also full rate because $N$ modulated symbols are transmitted for $N$ symbol time epochs.

It is noted that even though both the A-ST-CR and ST-CR codes achieve same diversity order $N$ with a given $\boldsymbol{\Theta}$, the A-ST-CR code enjoys larger coding gain than the ST-CR code [10]. This is mainly due to the fact that the A-ST-CR code can transmit the precoded symbols $r_{i}$ through the Alamouti encoder more reliably than the ST-CR code. From a this point of view, the new code of (7) has still room for increasing coding gain because the last precoded symbol pair $\left(r_{N-1}, r_{N}\right)$ is transmitted on only an $(N-1)$ th transmit antenna, not through the Alamouti encoder. Hence, by intuition, we may design a new code different from (7) where the second column vector of $\mathbf{S}_{\mathrm{A}}\left(r_{N-1}, r_{N}\right)$ is transmitted on the $(N-2)$ th antenna as follow:

$$
\mathbf{S}(\mathbf{r})=\frac{1}{\sqrt{2}}\left[\begin{array}{ccccc}
\mathbf{S}_{\mathrm{A}}\left(r_{1}, r_{2}\right) & \cdots & \mathbf{0}_{2 \times 1} & \mathbf{0}_{2 \times 1} & \mathbf{0}_{2 \times 1} \\
\vdots & \ddots & \vdots & \vdots & \vdots \\
\mathbf{0}_{2 \times 1}^{T} & \cdots & r_{N-3} & r_{N-2} & 0 \\
\mathbf{0}_{2 \times 1}^{T} & \cdots & -r_{N-2}^{*} & r_{N-3}^{*} & 0 \\
\mathbf{0}_{2 \times 1}^{T} & \cdots & 0 & r_{N} & r_{N-1} \\
\mathbf{0}_{2 \times 1}^{T} & \cdots & 0 & r_{N-1}^{*} & -r_{N}^{*}
\end{array}\right]
$$


where $1 / \sqrt{2}$ is a normalizing factor with the power constraint of $E\left\{\|\mathbf{S}(\mathbf{r})\|^{2}\right\}=$ $N$. For example, when using three transmit antennas, the code of (9) is given as

$$
\mathbf{S}(\mathbf{r})=\frac{1}{\sqrt{2}}\left[\begin{array}{ccc}
r_{1} & r_{2} & 0 \\
-r_{2}^{*} & r_{1}^{*} & 0 \\
0 & r_{4} & r_{3} \\
0 & r_{3}^{*} & -r_{4}^{*}
\end{array}\right] .
$$

Clearly, the code of (9) is full rate because $T_{0}=L$ to be $N$. This code is also guaranteed to achieve full spatial diversity like the new code of (7) because for any two distinct input vectors $\mathbf{x}, \mathbf{x}^{\prime}$,

$$
\begin{aligned}
\operatorname{det}(\mathbf{A})= & \left(\frac{1}{2}\right)^{N-1}\left(\prod_{i=1}^{\frac{N}{2}-2}\left(\left|d_{2 i-1}\right|^{2}+\left|d_{2 i}\right|^{2}\right)^{2}\right)\left(\left|d_{N-3}\right|^{2}+\left|d_{N-2}\right|^{2}\right) \times \\
& \left(\left|d_{N-3}\right|^{2}+\left|d_{N-2}\right|^{2}+\left|d_{N-1}\right|^{2}+\left|d_{N}\right|^{2}\right)\left(\left|d_{N-1}\right|^{2}+\left|d_{N}\right|^{2}\right)>0
\end{aligned}
$$

Even though both the proposed codes of (7) and (9) achieve full spatial diversity $N-1$ with a given $\Theta$, the code of (9) outperforms the one of (7) for all SNR values, which will be shown by computer simulations in Section IV.

\subsection{New STBC Using $\Theta$ of $N / 2 \times N / 2$}

As commented in the previous subsection, both the new codes of (7) and (9) using $\Theta$ of size $N \times N$ have some advantages of achieving full rate and full diversity for general QAM. However, these codes have a great drawback of not satisfying the Tarokh's orthogonal designs [2], leading to a much higher increase in ML decoding complexity at the receiver than the O-STBCs. Hence, in this subsection, by using a linear precoder $\Theta$ of size $N / 2 \times N / 2$, a new full rate and full diversity STBC possessing quasi-orthogonal property will be presented for any odd $N-1$ transmit antennas.

The proposed encoder first divides an input vector $\mathbf{x}$ of length $N$ into two input sub-vectors $\mathbf{x}_{i}=\left[x_{i, 1}, \cdots, x_{i, N / 2}\right]^{T}, i=1,2$ of length $N / 2$, i.e., $\mathbf{x}=\left[\mathbf{x}_{1}, \mathbf{x}_{2}\right]^{T}$. Each of these sub-vectors, $\mathbf{x}_{i}$ is then multiplied separately by a same linear precoder $\boldsymbol{\Theta}$ of size $N / 2 \times N / 2$, resulting in two precoded sub-vectors of length $N / 2, \mathbf{r}_{i}=\left[r_{i, 1}, \cdots, r_{i, N / 2}\right]^{T}=\boldsymbol{\Theta} \mathbf{x}_{i}, i=1,2$. Then, by serially grouping the $i$ th elements in both $\mathbf{r}_{1}$ and $\mathbf{r}_{2}$, total $N / 2$ precoded symbol pairs $\left(r_{1, i}, r_{2, i}\right)$, $i=1, \cdots, N / 2$ are generated. These pairs $\left(r_{1, i}, r_{2, i}\right)$ are then encoded independently by the Alamouti encoder [3] and transmitted on $N-1$ transmit antennas like the new code of (9)

$$
\mathbf{S}(\mathbf{r})=\frac{1}{\sqrt{2}}\left[\begin{array}{ccccc}
\mathbf{S}_{\mathrm{A}}\left(r_{1,1}, r_{2,1}\right) & \cdots & \mathbf{0}_{2 \times 1} & \mathbf{0}_{2 \times 1} & \mathbf{0}_{2 \times 1} \\
\vdots & \ddots & \vdots & \vdots & \vdots \\
\mathbf{0}_{2 \times 1}^{T} & \cdots & r_{1, \frac{N}{2}-1} & r_{2, \frac{N}{2}-1} & 0 \\
\mathbf{0}_{2 \times 1}^{T} & \cdots & -r_{2, \frac{N}{2}-1}^{*} & r_{1, \frac{N}{2}-1}^{*} & 0 \\
\mathbf{0}_{2 \times 1}^{T} & \cdots & 0 & r_{2, \frac{N}{2}} & r_{1, \frac{N}{2}} \\
\mathbf{0}_{2 \times 1}^{T} & \cdots & 0 & r_{1, \frac{N}{2}}^{*} & -r_{2, \frac{N}{2}}^{*}
\end{array}\right] .
$$


It is clear that this code is full-rate because $T_{0}=L$ to be $N$ as the code of (9). For the code of (12), we can easily calculate the determinant of the $\mathbf{A}$ matrix for any distinct input vectors $\mathbf{x}, \mathbf{x}^{\prime}$ as

$$
\begin{gathered}
\operatorname{det}(\mathbf{A})=\left(\frac{1}{2}\right)^{N-1}\left(\prod_{i=1}^{\frac{N}{2}-2}\left(\left|d_{1, i}\right|^{2}+\left|d_{2, i}\right|^{2}\right)^{2}\right)\left(\left|d_{1, \frac{N}{2}-1}\right|^{2}+\left|d_{2, \frac{N}{2}-1}\right|^{2}\right) \times \\
\left(\left|d_{1, \frac{N}{2}-1}\right|^{2}+\left|d_{2, \frac{N}{2}-1}\right|^{2}+\left|d_{1, \frac{N}{2}}\right|^{2}+\left|d_{2, \frac{N}{2}}\right|^{2}\right)\left(\left|d_{1, \frac{N}{2}}\right|^{2}+\left|d_{2, \frac{N}{2}}\right|^{2}\right)
\end{gathered}
$$

with $d_{i, j}=r_{i, j}-r_{i, j}^{\prime}$ where $r_{i, j}$ and $r_{i, j}^{\prime}$ represent the $j$ th elements of $\mathbf{r}_{i}$ and $\mathbf{r}_{i}^{\prime}$, respectively.

Indeed, for any two distinct vectors $\mathbf{x}, \mathbf{x}^{\prime}$, there exists at least one sub-vector pair $\left(\mathbf{x}_{i}, \mathbf{x}_{i}^{\prime}\right)$ satisfying $\mathbf{x}_{i} \neq \mathbf{x}_{i}^{\prime}$. This implies $r_{i, j} \neq r_{i, j}^{\prime}\left(\right.$ or $\left.d_{i, j} \neq 0\right),{ }^{\forall} j$, for a given index $i$ because of the rotation property of $\boldsymbol{\Theta}$. Hence, the determinant of (13) is always positive and thus, the code of (12) enjoys full spatial diversity of order $N-1$ like the new codes of (7) and (9). This code also satisfies the quasiorthogonal property like the conventional quasi-orthogonal STBCs (QO-STBCs) [4]-[6], allowing a ML decoder at the receiver to decode the two sub-vectors $\mathbf{x}_{1}$, $\mathbf{x}_{2}$, independently, which will be derived in the following.

First, by complex-conjugating the all elements of even indices in $\mathbf{y}$, denoted as $\mathbf{y}^{\prime}$, we can easily rearrange the ML metric given in (2) for the code of (12) as follow:

$$
\|\mathbf{y}-\mathbf{S}(\mathbf{x}) \mathbf{h}\|=\left\|\mathbf{y}^{\prime}-\mathbf{H}\left[\begin{array}{cc}
\Theta & \mathbf{0}_{\frac{N}{2}} \\
\mathbf{0}_{\frac{N}{2}} & \Theta^{2}
\end{array}\right] \mathbf{x}\right\|
$$

with

$$
\mathbf{H} \triangleq \frac{1}{\sqrt{2}}\left[\begin{array}{c}
\mathbf{h}_{1, \frac{N}{2}+1}\left(h_{1}, h_{2}\right) \\
\vdots \\
\mathbf{h}_{\frac{N}{2}-1, N-1}\left(h_{N-3}, h_{N-2}\right) \\
\mathbf{h}_{\frac{N}{2}, N}\left(h_{N-1}, h_{N-2}\right)
\end{array}\right]
$$

where $\mathbf{h}_{i, j}(a, b)$ denotes an $2 \times N$ matrix whose $i$ th and $j$ th column vectors are $\left[a, b^{*}\right]^{T}$ and $\left[b,-a^{*}\right]^{T}$, respectively, and all other column vectors with zero elements. The equality of (14) uses the fact that the conjugating of any number of elements in a vector preserves the magnitude of the vector. At this point of time, we will define an unitary matrix $\mathbf{B}$ generated by appropriately normalizing the elements in $\mathbf{H}$ of (15), given as

$$
\mathbf{B} \triangleq\left[\begin{array}{c}
\mathbf{h}_{1, \frac{N}{2}+1}\left(h_{1}, h_{2}\right) / \rho\left(h_{1}, h_{2}\right) \\
\vdots \\
\mathbf{h}_{\frac{N}{2}-1, N-1}\left(h_{N-3}, h_{N-2}\right) / \rho\left(h_{N-3}, h_{N-2}\right) \\
\mathbf{h}_{\frac{N}{2}, N}\left(h_{N-1}, h_{N-2}\right) / \rho\left(h_{N-1}, h_{N-2}\right)
\end{array}\right]
$$


where $\rho(a, b) \triangleq \sqrt{|a|^{2}+|b|^{2}}$. Note that the matrix $\mathbf{B}$ of (16) satisfies the unitarity, i.e., $\mathbf{B}^{*} \mathbf{B}=\mathbf{I}_{N}$ where $\mathbf{I}_{N}$ represents an identity matrix of size $N \times N$.

Then by using the complex conjugate of $\mathbf{B}$ of (16), the ML metric in the right side of (14) can be decomposed into two functions composed of $\mathbf{x}_{1}$ and $\mathbf{x}_{2}$, respectively, as follows:

$$
\begin{aligned}
\left\|\mathbf{y}^{\prime}-\mathbf{H}\left[\begin{array}{cc}
\boldsymbol{\Theta} & \mathbf{0}_{\frac{N}{2}} \\
\mathbf{0}_{\frac{N}{2}} & \boldsymbol{\Theta}
\end{array}\right] \mathbf{x}\right\| & =\left\|\mathbf{B}^{*} \mathbf{y}^{\prime}-\mathbf{B}^{*} \mathbf{H}\left[\begin{array}{cc}
\boldsymbol{\Theta} & \mathbf{0}_{\frac{N}{2}} \\
\mathbf{0}_{\frac{N}{2}} & \boldsymbol{\Theta}
\end{array}\right] \mathbf{x}\right\| \\
& =\left\|\left[\begin{array}{l}
\mathbf{z}_{1} \\
\mathbf{z}_{2}
\end{array}\right]-\left[\begin{array}{cc}
\boldsymbol{\Lambda} & \mathbf{0}_{\frac{N}{2}} \\
\mathbf{0}_{\frac{N}{2}} & \boldsymbol{\Lambda}
\end{array}\right]\left[\begin{array}{cc}
\boldsymbol{\Theta} & \mathbf{0}_{\frac{N}{2}} \\
\mathbf{0}_{\frac{N}{2}} & \boldsymbol{\Theta}
\end{array}\right]\left[\begin{array}{l}
\mathbf{x}_{1} \\
\mathbf{x}_{2}
\end{array}\right]\right\| \\
& =\sum_{i=1}^{2}\left\|\mathbf{z}_{i}-\boldsymbol{\Lambda} \boldsymbol{\Theta} \mathbf{x}_{i}\right\|
\end{aligned}
$$

where $\left[\mathbf{z}_{1}, \mathbf{z}_{2}\right]^{T}=\mathbf{B}^{*} \mathbf{y}^{\prime}$ with $N / 2$ dimensional vectors $\mathbf{z}_{1}, \mathbf{z}_{2}$ and

$$
\boldsymbol{\Lambda} \triangleq \frac{1}{\sqrt{2}} \operatorname{diag}\left(\rho\left(h_{1}, h_{2}\right), \cdots, \rho\left(h_{N-3}, h_{N-2}\right), \rho\left(h_{N-1}, h_{N-2}\right)\right) .
$$

The equality of (17) uses the unitarity of the matrix $\mathbf{B}$ preserving the energy of a vector. Hence, we can easily know that the minimization of the ML metric in the left of (17) is equivalent to the minimization of the two equations of $\mathbf{x}_{1}$ and $\mathbf{x}_{2}$ in (19), separately. Thus, the ML receiver for the code of (12) can decode the input sub-vectors $\mathbf{x}_{i}$, independently, by choosing $\hat{\mathbf{x}}_{i}$ such that

$$
\hat{\mathbf{x}}_{i}=\arg \min _{\mathbf{x}_{i}}\left\|\mathbf{z}_{i}-\boldsymbol{\Lambda} \boldsymbol{\Theta} \mathbf{x}_{i}\right\|, \quad i=1,2 .
$$

\section{Simulation Results}

All of STBCs considered in this Section are assumed to be ones with $N_{t}$ transmit and one receive antennas over quasi-static Rayleigh fading channels. Also, it is assumed that the fading channel gains are perfectly known at the receiver. With these assumptions, we provide the simulation results of the three proposed codes of (7), (9) and (12) with $N_{t}=3,5$ for QPSK and $N_{t}=3$ for 16-QAM. The codes of (17) and (9) use the same unitary precoders $\Theta$ of (5) of size $4 \times 4$ for $N_{t}=3$ and of size $6 \times 6$ with $P=36$ for $N_{t}=5$, respectively. Also, the quasi-orthogonal code of (12) uses $\Theta$ of size $2 \times 2$ constructed using (5) for $N_{t}=3$ and $\Theta$ given in (6) for $N_{t}=5$. For the comparison of performances, the results of the ST-CR code [9], [10] in (3) using $\boldsymbol{\Theta}$ of (5) and the Alamouti scheme [3] are also included. Furthermore, we include the results of the maximal ratio receiver combining (MRRC) scheme using appropriately normalized one transmit and $N_{t}$ receive antennas [3].

Figs. 2 and 3 show the average symbol error rate (SER) curves versus $E_{s} / N_{0}$ for QPSK and 16-QAM, respectively. From these results, we see that all of the 
proposed codes achieve full spatial diversity and also, larger coding gains than the existing ST-CR code [8], [9] for all SNR values. This is because the proposed codes can transmit the precoded symbols $r_{i}$ more reliably than the conventional ST-CR code by using the Alamouti encoder with two transmit antennas. In particular, the code of (9) is shown to achieve the best performance among the three proposed codes for all considered modulations and transmit antennas. For the case of QPSK, this code enjoys approximately 1.5dB larger coding gain than the existing ST-CR code for $N_{t}=3,5$ at average $\mathrm{SER}=10^{-5}$ and for the case of 16-QAM, $3 \mathrm{~dB}$ for $N_{t}=3$. Also, we notice that the best code exhibits error performance within only about $1 \mathrm{~dB}$ of the MRRC scheme.

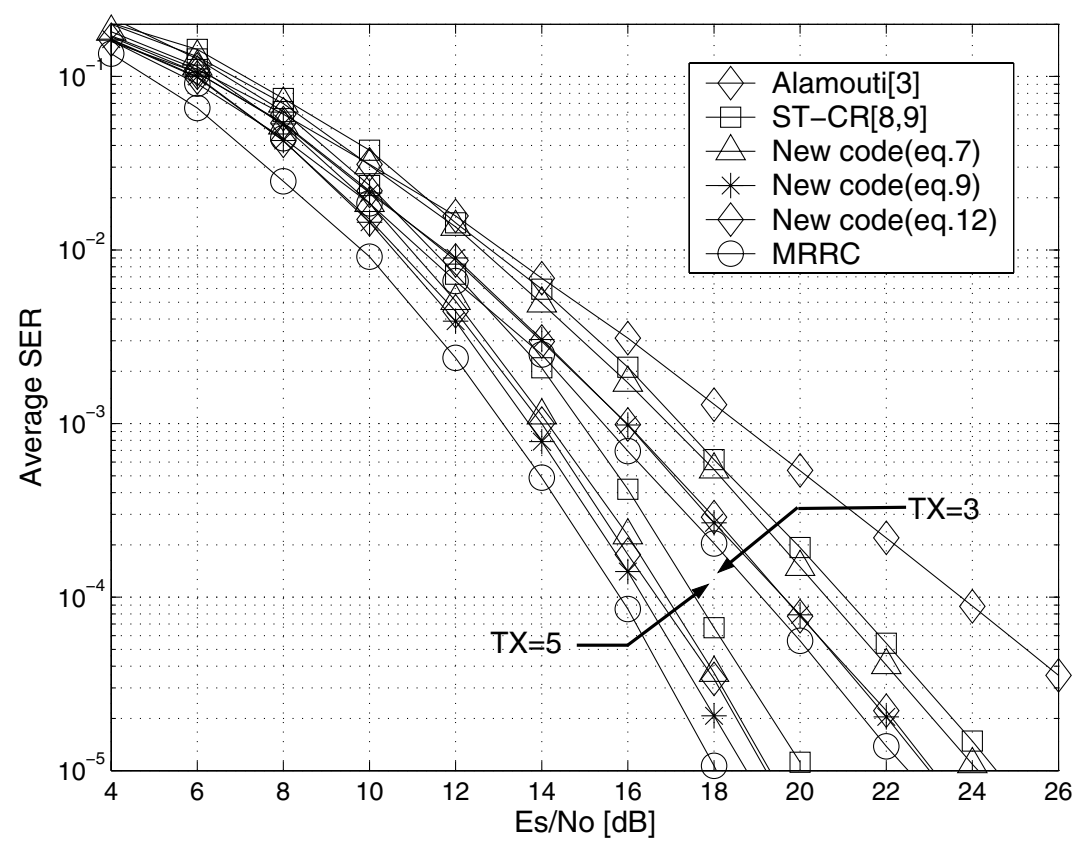

Fig. 2. Average SER versus $E_{s} / N_{0}[\mathrm{~dB}]$ for QPSK

We also know from these results that the proposed quasi-orthogonal code of (12) exhibits approximately same error performance as the best code of (9) for all SNR values for considered modulations and transmit antennas. It is noted that this code satisfies the quasi-orthogonal characteristic like the conventional QOSTBCs [4]-[6], leading to greatly simplified ML decoding at the receiver. Hence, considering both the performance results and the decoding complexity, this code may be a promising solution for the next generation mobile communications. 


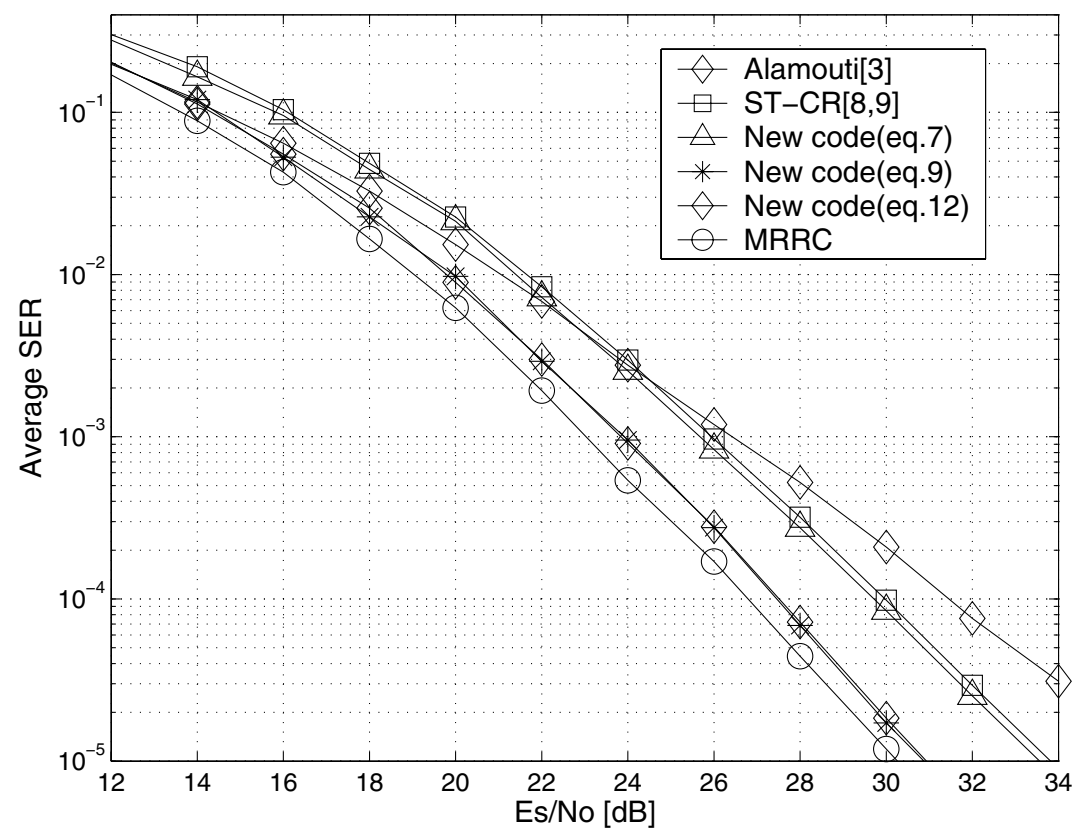

Fig. 3. Average SER versus $E_{s} / N_{0}[\mathrm{~dB}]$ for 16-QAM and three transmit antennas

\section{Conclusions}

In this paper, we proposed new STBCs achieving full rate and full diversity for QAM and quasi-static Rayleigh fading channels when using any odd number of transmit antennas. These codes are designed by serially concatenating the constellation-rotating precoders with the Alamouti scheme like the conventional A-ST-CR code. We showed by computer simulations that all of proposed codes outperform the existing ST-CR codes for any considered modulations and transmit antennas. Particularly, the codes possessing quasi-orthogonal characteristic are also included in this class, allowing simple ML decoding with virtually no performance loss compared to the best code in the class.

\section{References}

1. V. Tarokh, N. Seshadri, and A. R. Calderbank, "Space-time codes for high data rate wireless communication: performance criterion and code construction," IEEE Trans. Inform. Theory, vol. 44, no. 2, pp. 744-765, Mar. 1998.

2. V. Tarokh, H. Jafarkhani, and A. R. Calderbank, "Space-time block coding for wireless communications: Theory of generalized orthogonal designs," IEEE Trans. Inform. Theory, vol. 45, pp. 1456-1467, July 1999. 
3. S. M. Alamouti, "A simple transmit diversity technique for wireless communications," IEEE J. Select. Areas Commun., vol. 16, no. 8, pp. 1451-1458, Oct. 1998.

4. H. Jafarkhani, "A quasi-orthogonal space-time block code," IEEE Trans. Commun., vol. 49, pp. 1-4, Jan. 2001.

5. N. Sharma and C. B. Papadias, "Improved quasi-orthogonal codes through constellation rotation," IEEE Trans. Commun., vol. 52, pp. 332-335, Mar. 2003.

6. W. Su and X.-G. Xia, "Signal constellations for quasi-orthogonal space-time block codes with full diversity," IEEE Trans. Inform. Theory, vol. 50, pp. 2331-2347, Oct. 2004.

7. X. Giraud and E. Boutillon, and J. C. Belfore, "Algebraic tools to build modulation schemes for fading channels," In IEEE Trans. Inform. Theory, vol. 43, pp. 938-952, May 1997.

8. Y. Xin, Z. Wang, and G. B. Giannakis, "Space-time constellation-rotating codes maximizing diversity and coding gains," in Proc. GLOBECOM, vol. 1, pp. 455-459, 2001.

9. Y. Xin, Z. Wang, and G. B. Giannakis, "Space-time diversity systems based on linear constellation precoding," IEEE Tran. Wireless Commun. vol. 2, pp. 294-309, Mar. 2003.

10. T. Jung and K. Cheun, "Design of concatenated space-time block codes using signal space diversity and the Alamouti scheme," IEEE Commun. Lett., vol. 7, pp. 329-331, July 2003. 